\title{
ON POSITIVE HARMONIC POLYNOMIALS ${ }^{1}$
}

\author{
To Hans Rademacher, in friendship
}

BY

\section{Gabor Szegö}

\section{Introduction}

1. In 1915, Georg Pick [2] made the following observation. Let $u(x, y, z)$ belong to the class $K$ of all harmonic functions which are not identically zero, moreover regular and nonnegative in the unit sphere $x^{2}+y^{2}+z^{2}<1$. We expand $u$ in a series of spherical harmonics:

$$
u(x, y, z)=\sum_{n=0}^{\infty} H_{n}(x, y, z),
$$

where $H_{n}$ is a homogeneous harmonic polynomial of degree $n$. We have then for $x^{2}+y^{2}+z^{2} \leqq 1$,

$$
\left|H_{1} / H_{0}\right| \leqq 3 \text {. }
$$

The constant 3 is the best possible one. Of course, $H_{0}=u(0,0,0)>0$.

Inequality (1.2) can be proved and generalized easily with the aid of the well-known representation (cf. [1, p. 444]):

$$
H_{n}(x, y, z)=\frac{2 n+1}{4 \pi} r^{n} \iint u\left(x^{\prime}, y^{\prime}, z^{\prime}\right) P_{n}(\cos \gamma) d \sigma^{\prime} .
$$

Here we use the polar equivalent $r, \theta, \phi$ of the cartesian coordinates $x, y, z$. The integration refers to $x^{\prime}, y^{\prime}, z^{\prime}$ or $1, \theta^{\prime}, \phi^{\prime}$, and $\gamma$ denotes the spherical distance of the points $1, \theta, \phi$ and $1, \theta^{\prime}, \phi^{\prime}$ on the unit sphere; $d \sigma^{\prime}$ is the surface element at $1, \theta^{\prime}, \phi^{\prime}$, and $P_{n}$ denotes Legendre's polynomial. We find from

$$
\left|H_{n} / H_{0}\right| \leqq 2 n+1
$$

The bound is the best possible and is attained for the function

$$
u(x, y, z)=\frac{1-r^{2}}{\left(1-2 r \cos \gamma+r^{2}\right)^{3 / 2}}=\sum_{n=0}^{\infty}(2 n+1) P_{n}(\cos \gamma) r^{n} .
$$

2. In 1927, I discussed [3] the following refinement of Pick's inequality. Let $N$ be a nonnegative integer; we denote by $K_{N}$ the class of finite (terminating) harmonic polynomials

$$
u(x, y, z)=\sum_{n=0}^{N} H_{n}(x, y, z),
$$

which are again not identically zero and nonnegative in the unit sphere. The

Received June 26, 1961.

1 This research was supported by the National Science Foundation. 
refinement of $(1.2)$ is then:

$$
\left|H_{1} / H_{0}\right| \leqq 3 \rho_{N},
$$

where $\rho_{N}$ is the largest zero of the Legendre polynomial $P_{q+1}(t)$ if $N=2 q$ is even, and the largest zero of the polynomial $P_{q+1}(t)+P_{q+2}(t)$ if $N=2 q+1$ is odd. In both cases, the bound $3 \rho_{N}$ is sharp, i.e., attained for a suitable member of the class $K_{N}$ at a suitable point $x, y, z$ of the unit sphere. The exact range of the quotient $H_{1} / H_{0}$ as $u$ is arbitrary in $K_{N}$ and $x, y, z$ is arbitrary in the unit sphere, is the closed interval $\left[-3 \rho_{N}, 3 \rho_{N}\right]$.

3. The purpose of the present note is to deal with the corresponding problem for the quotient $H_{2} / H_{0}$ referring again to the class $K_{N}$. Thus we aim at a refinement of the inequality (1.4) in the special case $n=2$. This case can be treated by methods essentially similar to those used for $n=1$. The nature of the result is however slightly different since for $n=2$ the range of the quotient in question is a nonsymmetrical interval around the origin. The reason for this discrepancy is that the range of Legendre's polynomial $P_{n}(x)$ in $-1 \leqq x \leqq 1$ is the symmetrical interval $[-1,+1]$ if $n$ is odd, and a certain nonsymmetrical interval $\left[-\mu_{n},+1\right], 0<\mu_{n}<1$, if $n$ is even.

We shall prove the following

THeonem. The range of the quotient $H_{2} / H_{0}$, as $u$ runs over the class $K_{N}$ and $(x, y, z)$ runs in the unit sphere, is the interval

$$
5 \cdot\left(3 t^{2}-1\right) / 2 \leqq H_{2} / H_{0} \leqq 5 \cdot\left(3 t^{\prime 2}-1\right) / 2,
$$

where $t^{\prime}$ and $t^{\prime \prime}$ denote the smallest and largest positive zero of $P_{[N / 2]+2}(t)$, $0<t^{\prime}<t^{\prime \prime}<1$, respectively, provided [N/2] is even. The meaning of $t^{\prime \prime}$ is still the same if $[N / 2]$ is odd, but $t^{\prime}$ will be in the latter case the smallest positive zero of the derivative $P_{[N / 2]+2}^{\prime}(t)$.

The general case, i.e., the corresponding refinement of (1.4) for the class $K_{N}$ for an arbitrary $n, n \geqq 3$, seems to be rather difficult. There is, however, no difficulty in determining the exact range of the quotient $H_{n} / H_{0}$ for arbitrary $n$ if $u \subset K$. This is the interval $\left[-\mu_{n}(2 n+1), 2 n+1\right]$ where $-\mu_{n}$ is the minimum of Legendre's polynomial $P_{n}(t)$ for $-1<t<1$. This minimum is in fact $=-1$ if $n$ is odd, and it is the first relative minimum of $P_{n}(t)$ from the right end point of the interval $-1<t<1$ if $n$ is even (cf. [4, Theorem 7.3.1, p. 162]). The quantity $\mu_{n}$ is decreasing with increasing $n, n$ even, [5] and converges for $n \rightarrow \infty$ to minus the absolute minimum of the Bessel function $J_{0}(x)$ which is 0.4028 .

4. As in the proof of (1.7), we shall make use of an important theorem of F. Lukács on polynomials $f(t)$ which are nonnegative in the real interval $a \leqq t \leqq b$. If the degree of $f(t)$ is $n$, we have the following representation:

$$
f(t)=[A(t)]^{2}+(t-a)(b-t)[B(t)]^{2} \quad \text { if } n \text { is even, } n=2 q,
$$


and

$$
f(t)=(t-a)[C(t)]^{2}+(b-t)[D(t)]^{2} \quad \text { if } n \text { is odd, } n=2 q+1 .
$$

Here $A, B, C, D$ are suitable polynomials of $t$ whose degrees do not exceed $q, q-1, q, q$, respectively.

For the proof of this theorem, cf. for instance [4, p. 4].

\section{Symmetrization. Reduction of the problem}

1. For the proof of the theorem formulated above, we observe first that in view of the extremum principle it is sufficient to consider only the points on the unit sphere $x^{2}+y^{2}+z^{2}=1$. Also, any rotation of the unit sphere leaves the class $K_{N}$ unchanged; thus it is sufficient to establish the range of the values of $H_{2} / H_{0}$ for $x=y=0, z=1$, i.e., for $r=1, \theta=0$. Now for every $n$

$$
H_{n}(0,0,1)=\frac{2 n+1}{4 \pi} \iint u\left(x^{\prime}, y^{\prime}, z^{\prime}\right) P_{n}\left(\cos \theta^{\prime}\right) \sin \theta^{\prime} d \theta^{\prime} d \phi^{\prime},
$$

where $\left(x^{\prime}, y^{\prime}, z^{\prime}\right)=\left(1, \theta^{\prime}, \phi^{\prime}\right)$. Using the standard representation of the surface harmonics (cf. [1, p. 444]), we see that

$$
\frac{1}{2 \pi} \int_{0}^{2 \pi} u\left(x^{\prime}, y^{\prime}, z^{\prime}\right) d \phi^{\prime}
$$

is a zonal harmonic polynomial of the form

$$
f\left(\cos \theta^{\prime}\right)=\sum_{n=0}^{N} a_{n} P_{n}\left(\cos \theta^{\prime}\right)
$$

where the coefficients $a_{n}$ are real. Obviously the associated solid harmonic $\sum_{n=0}^{N} a_{n} r^{n} P_{n}\left(\cos \theta^{\prime}\right)$ belongs also to $K_{N}$, and the values $H_{N}(0,0,1)$ do not change when we pass from the original function $u(x, y, z)$ to the "symmetrized" zonal function (2.2).

Thus without impairing the generality we are led to the following reduced

Problem. Let $f(t)=\sum_{n=0}^{N} a_{n} P_{n}(t)$ denote an arbitrary polynomial of degree $N$ which is not identically zero and moreover nonnegative in the interval $-1 \leqq t \leqq 1$. We have to determine the range of the ratio $a_{2} / a_{0}$.

2. The expression

$$
(f(t)+f(-t)) / 2=g\left(t^{2}\right)=g(\tau)
$$

represents a polynomial in $t^{2}=\tau$ of degree [N/2], and

$$
\begin{aligned}
& a_{0}=\frac{1}{2} \int_{-1}^{+1} f(t) d t=\frac{1}{2} \int_{0}^{1} g(\tau) \tau^{-1 / 2} d \tau, \\
& a_{2}=\frac{5}{2} \int_{-1}^{+1} f(t) P_{2}(t) d t=\frac{5}{2} \int_{0}^{1} g(\tau) \frac{3 \tau-1}{2} \tau^{-1 / 2} d \tau,
\end{aligned}
$$


so that

$$
\frac{a_{2}}{a_{0}}=5 \int_{0}^{1} g(\tau) \frac{3 \tau-1}{2} \tau^{-1 / 2} d \tau: \int_{0}^{1} g(\tau) \tau^{-1 / 2} d \tau .
$$

Here $g(\tau)$ is an arbitrary polynomial of degree [N/2] which is not identically zero and moreover nonnegative in the interval $0 \leqq \tau \leqq 1$.

3. Now we apply the representation described in $\$ 1.4$ :

$$
\begin{array}{ll}
g(\tau)=[A(\tau)]^{2}+\tau(1-\tau)[B(\tau)]^{2} & \text { if }[N / 2] \text { is even, }[N / 2]=2 q, \\
g(\tau)=\tau[C(\tau)]^{2}+(1-\tau)[D(\tau)]^{2} & \text { if }[N / 2] \text { is odd, }[N / 2]=2 q+1
\end{array}
$$

The degrees of the polynomials $A, B, C, D$ are $q, q-1, q, q$, respectively. We seek the range of the quotients:

$$
\begin{aligned}
\int_{0}^{1}[A(\tau)]^{2} \frac{3 \tau-1}{2} \tau^{-1 / 2} d \tau & : \int_{0}^{1}[A(\tau)]^{2} \tau^{-1 / 2} d \tau \\
\int_{0}^{1} \tau(1-\tau)[B(\tau)]^{2} \frac{3 \tau-1}{2} \tau^{-1 / 2} d \tau & : \int_{0}^{1} \tau(1-\tau)[B(\tau)]^{2} \tau^{-1 / 2} d \tau \\
\int_{0}^{1} \tau[C(\tau)]^{2} \frac{3 \tau-1}{2} \tau^{-1 / 2} d \tau & : \int_{0}^{1} \tau[C(\tau)]^{2} \tau^{-1 / 2} d \tau \\
\int_{0}^{1}(1-\tau)[D(\tau)]^{2} \frac{3 \tau-1}{2} \tau^{-1 / 2} d \tau & : \int_{0}^{1}(1-\tau)[D(\tau)]^{2} \tau^{-1 / 2} d \tau
\end{aligned}
$$

In $\$ 3$ we shall prove that the second range is contained in the first one. Since in (2.5) and (2.6) we can choose $B(\tau) \equiv 0$, the range of $(2.5)$ for $[N / 2]$ even, will be identical with the range of the first quotient in (2.7). A similar reasoning applies in the case when $[N / 2]$ is odd.

\section{Mechanical quadrature}

1. To the integrals occurring in (2.7) we apply the formula of GaussJacobi [4, p. 47]. Let $w(\tau)$ be an arbitrary weight function in the interval $0 \leqq \tau \leqq 1, p_{m}(\tau)$ the associated orthogonal polynomial of degree $m, \tau_{v}$ the zeros of $p_{m}(\tau), \lambda_{v}$ the corresponding Christoffel numbers, $1 \leqq v \leqq m$, $0<\tau_{v}<1, \lambda_{v}>0$. We have then

$$
\begin{aligned}
\int_{0}^{1}[S(\tau)]^{2} \frac{3 \tau-1}{2} w(\tau) d \tau: & \int_{0}^{1}[S(\tau)]^{2} w(\tau) d \tau \\
& =\sum_{v=1}^{m} \lambda_{v}\left[S\left(\tau_{v}\right)\right]^{2} \frac{3 \tau_{v}-1}{2}: \sum_{v=1}^{m} \lambda_{v}\left[S\left(\tau_{v}\right)\right]^{2},
\end{aligned}
$$

where $S(\tau)$ is any polynomial of degree $s$; these formulas hold provided $2 s+1 \leqq 2 m-1, s \leqq m-1$. If $s$ is given, we choose $m=s+1$.

From the representation (3.1), we conclude that the range of the ratio in (3.1), when $S(\tau)$ runs over all polynomials not identically zero and of degree 
$s$, is the closed interval

$$
\left[\left(3 \tau^{\prime}-1\right) / 2,\left(3 \tau^{\prime \prime}-1\right) / 2\right],
$$

where $\tau^{\prime}$ and $\tau^{\prime \prime}$ denote the smallest and largest zero of $p_{m}(\tau)=p_{s+1}(\tau)$, respectively. Indeed, by prescribing the values of the polynomial $S(\tau)$ at $m=s+1$ points, it will be uniquely determined. If the "data" are not all zero, $S(\tau)$ will be not identically zero. We use the fact that $(3 \tau-1) / 2$ is monotonic for $0<\tau<1$.

In (2.7), we have

$$
\begin{aligned}
w(\tau) & =\tau^{-1 / 2}, \tau^{1 / 2}(1-\tau), \tau^{1 / 2}, \tau^{-1 / 2}(1-\tau), \\
s & =q, q-1, q, q \\
m & =q+1, q, q+1, q+1
\end{aligned}
$$

respectively.

2. Transforming the interval $[0,1]$ into the interval $[-1,1]$, we obtain the following Jacobi polynomials corresponding to the cases (3.3) (notation as in [4, Chapter IV]):

$$
P_{q+1}^{(0,-1 / 2)}(2 \tau-1), \quad P_{q}^{(1,1 / 2)}(2 \tau-1), \quad P_{q+1}^{(0,1 / 2)}(2 \tau-1), \quad P_{q+1}^{(1,-1 / 2)}(2 \tau-1) .
$$

Substituting $\tau=t^{2}$ and taking [4, (4.1.5), p. 59] into account, we find the following polynomials:

$$
P_{2 q+2}(t), \quad t^{-1} P_{2 q+1}^{(1,1)}(t), \quad t^{-1} P_{2 q+3}(t), \quad P_{2 q+2}^{(1,1)}(t),
$$

where $P_{n}$ is Legendre's polynomial and $P_{n}^{(1,1)}(t)$ denotes the Jacobi polynomial associated with the weight function $1-t^{2}$ in $[-1,1]$. We remark that $P_{n}^{(1,1)}(t)$ is, except for a constantfactor, identical with $P_{n+1}^{\prime}(t)$ (derivative of $P_{n+1}(t)$; cf. [4, (4.21.7), p. 63]).

3. Let $[N / 2]=2 q$. Since the second function in (3.4) is, except for the factor $t^{-1}$, the derivative of the first one, the largest zero of the first function will surpass that of the second function; a similar conclusion holds for the smallest positive zeros of these functions.

Let $[N / 2]=2 q+1$. The same as before holds for the largest zeros. But the smallest positive zero of $P_{2 q+3}^{\prime}(t)$ is less than the smallest positive zero of $P_{2 q+3}(t)$.

This establishes the assertion of the theorem announced in $\$ 1$.

4. As an example, we treat the special cases $N=1$ and $N=2$ or 3 . In the first case $a_{2}=0$, and indeed $P_{2}(t)$ has only one positive zero which makes the two quantities occurring in (1.8) vanish. In the second case $(N=2$ or 3$),[N / 2]=1$ is odd. The polynomial $P_{3}(t)=\frac{5}{2} t^{3}-\frac{3}{2} t$ has one positive zero $t^{\prime \prime}, t^{\prime \prime 2}=\frac{3}{5}$, and $P_{3}^{\prime}(t)=\frac{15}{2} t^{2}-\frac{3}{2}$ has one positive zero $t^{\prime}, t^{\prime 2}=\frac{1}{5}$. Thus 
the range (1.8) will be

$$
-1 \leqq a_{2} / a_{0} \leqq 2 .
$$

This can be seen directly from the representation

$$
\left(u_{0}+u_{1} t\right)^{2}+\left(1-t^{2}\right) u_{2}^{2}=a_{0} P_{0}(t)+a_{1} P_{1}(t)+a_{2} P_{2}(t) .
$$

We have

and

$$
\begin{aligned}
& a_{0}=u_{0}^{2}+\frac{1}{3} u_{1}^{2}+\frac{2}{3} u_{2}^{2}, \\
& a_{1}=2 u_{0} u_{1}, \\
& a_{2}=\frac{2}{3} u_{1}^{2}-\frac{2}{3} u_{2}^{2},
\end{aligned}
$$

$$
\left(\frac{2}{3} u_{1}^{2}-\frac{2}{3} u_{2}^{2}\right) /\left(u_{0}^{2}+\frac{1}{3} u_{1}^{2}+\frac{2}{3} u_{2}^{2}\right)
$$

has indeed the minimum -1 and the maximum 2 .

For $N=4$ we obtain by an easy but more elaborate calculation the following range:

$$
-1.633=(5-3 \sqrt{30}) / 7 \leqq a_{2} / a_{0} \leqq(5+3 \sqrt{30}) / 7=3.061 .
$$

\section{Bibliography}

1. R. Courant and D. Hilbert, Methoden der mathematischen Physik, vol. 1, 2nd ed., Berlin, 1931.

2. G. Prck, Ein Abschätzungssatz für positive Newtonsche Potentiale, Jber. Deutsch. Math. Verein., vol. 24 (1915), pp. 329-332.

3. G. SzEGö, Koeffizientenabschätzungen bei ebenen und räumlichen harmonischen Entwicklungen, Math. Ann., vol. 96 (1927), pp. 601-632.

4. - Orthogonal polynomials, Amer. Math. Soc. Colloquium Publications, vol. 23, rev. ed., New York, 1959.

5. - On the relative extrema of Legendre polynomials, Boll. Un. Mat. Ital. (3), vol. 5 (1950), pp. 120-121.

STANFORD UNIVERSITY

Stanford, California 